\title{
ASPECTOS EPIDEMIOLÓGICOS DE LA SEPSIS, EN UNIDADES DE CUIDADOS INTENSIVOS SANTA MARTA, COLOMBIA
}

\section{EPIDEMIOLOGICAL ASPECTS OF SEPSIS IN INTENSIVE CARE UNITS SANTA MARTA, COLOMBIA}

\section{TÍTULO CORTO: ASPECTOS EPIDEMIOLÓGICOS DE LA SEPSIS}

\author{
Yolima Pertuz-Meza ${ }^{1}$, Celenny Perez-Quinteroº ${ }^{2}$ Yadira Pabón-Varela ${ }^{3}$
}

Recibido en noviembre 03 de 2015

Aceptado en febrero 23 de 2016

\section{RESUMEN}

Determinar los aspectos epidemiológicos relacionados con sepsis en los pacientes atendidos en las unidades de cuidados intensivos de adultos de Santa Marta. Se realizó un estudio descriptivo transversal en el año 2014; muestreo aleatorio simple, en pacientes atendidos en unidades de cuidado intensivo adulto (UCIA) en Santa Marta. Se utilizó una muestra de 100 pacientes mayores de 18 años con diagnóstico de sepsis, las variables estudiadas fueron: edad, sexo, agente causal, principales focos de infección entre otros. Como resultado se encontró que la edad promedio de los casos fue de 63 , los principales microorganismos causales fueron las bacterias gram negativas con un $76 \%$, siendo la Echerichia coli, Klebsiella pneumoniae, Pseudomona aureuginosa y Serratia marcensens las más aisladas, en segundo lugar, las bacterias gram positivas con un 20\%, principalmente el Staphylococcus aureus, y por último los hongos con un $4 \%$, siendo Candida albicans y Candida haemulonii las levaduras causantes. Los focos infecciosos más frecuentes son; el abdominal (30\%), seguido del tracto urinario (22\%) y la piel y tejidos blandos (18\%). Se concluye que los principales agentes causales de sepsis son las bacterias gram negativas y el principal foco primario infeccioso es la sepsis de origen abdominal.

Palabras clave: Sepsis; UCI; epidemiología; adulto

\section{ABSTRACT}

Determine the epidemiological aspects of sepsis in patients treated in adult intensive care units in Santa Marta. A descriptive, cross-sectional study in 2014, simple random sampling, in patients seen in adult intensive care units (UCIA) in Santa Marta, a sample of 100 patients older than 18 years diagnosed with sepsis was used variables They studied were age, sex, causal agent, main sources of infection among others. As a result it was found that the

\footnotetext{
1. Bacterióloga, especialista en epidemiología, Docente, Universidad Cooperativa de Colombia, Colombia. Correo: yoliperme@yahoo.com; yolima.pertuz@campusucc.edu.co

2. Enfermera, Docente, Universidad Cooperativa de Colombia, Colombia. Correo: celenny.perezq@campusucc.edu.co

3. Enfermera, Docente, Universidad Cooperativa de Colombia, Colombia. Correo: Yadira.pabon@campusucc.edu.co
} 
average age of cases was 63 , the main causative organisms were gram-negative bacteria with $76 \%$, the Escherichia coli, Klebsiella pneumoniae, Pseudomonas and Serratia marcensens aureuginosa more isolated, secondly the Gram positive bacteria with $20 \%$, mainly Staphylococcus aureus, fungi and finally with $4 \%$ being Candida albicans and Candida haemulonii causing yeast. The most common sources of infection are; abdominal (30\%), followed by the urinary tract $(22 \%)$ and skin and soft tissue (18\%). It is concluded that the main causative agents are gram negative sepsis bacteria and the main infectious primary focus is the abdominal sepsis.

Keywords: sepsis; ICU; epidemiology; adult

\section{INTRODUCCIÓN}

$\mathrm{D}$ esde un punto de vista sanitario, la sepsis es muy relevante, la incidencia de la sepsis grave fue de 3 casos de cada 1.000 habitantes (751.000 casos / año para la población de Estados Unidos), superando la incidencia del adquirido síndrome de inmunodeficiencia (SIDA) y los tipos predominantes de cáncer, dando como resultado 215.000 muertes por año $(28,6 \% \text { de los casos })^{1}$. En España, la incidencia de la sepsis grave es 104 por 100.000 habitantes / año, con una tasa de mortalidad del 20,5\%, mientras que la incidencia de shock séptico es del 31 por 100.000 habitantes / año, con una tasa de mortalidad del 45,7\%. En América Latina se informan tasas de mortalidad más altas que en los países desarrollados y mayor frecuencia en la población menor de 50 años, lo que relacionan con factores de riesgo, así como la presencia de enfermedades infectocontagiosas ${ }^{2}$.

Cualquier microorganismo puede conducir a un cuadro séptico, pero con mayor frecuencia las bacterias ${ }^{3}$. La sepsis continúa siendo una afección frecuente que afecta a un $12-34 \%$ de pacientes ingresados en UCI (Unidad de Cuidado Intensivo) ${ }^{4}$.

Se ha determinado que las bacterias Gram negativas son las principales causantes, pero en la actualidad se han visto superadas por las bacterias Gram positivas. Las infecciones polimicrobianas y los hongos son causantes de un incremento en la incidencia de pacientes sépticos. Los virus y parásitos tienen menor porcentaje en los casos. Así mismo, en un gran número de pacientes con sepsis no se logra determinar el microorganismo causante ${ }^{5}$. El principal foco infeccioso asociado a sepsis es el de origen abdominal, seguido del pulmonar?
La sepsis es definida como el resultado de la respuesta sistémica del huésped ante un microorganismo, esta patología consiste en la invasión del cuerpo por microorganismos patógenos y la reacción que estos y sus toxinas provocan en sus tejidos causando daño $0^{3,6}$.

Esta enfermedad la clasifican en sepsis, sepsis severa y choque séptico de acuerdo el grado de afectación sistémica según las definiciones de la Conferencia Internacional de Sepsis de $2003^{7,8}$. Es así como un paciente séptico puede tener manifestaciones clínicas de infección más fiebre, taquicardia, taquipnea con número de leucocitos anormales, la sepsis severa es el paciente séptico que cursa con hipotensión, hipoxemia oliguria pero responde enseguida a los líquidos intravenosos y el choque séptico se caracteriza por una sepsis severa que dura más de una hora a pesar de la administración de líquidos intravenosos por lo que se requiero el uso de vasopresores $^{1,9,10}$.

En muchos pacientes las manifestaciones clínicas de la sepsis varían sobre todo si se trata de inmunodeprimidos. Además de los hemocultivos se recomienda el uso de biomarcadores de inflamación e infección en urgencias que dan a conocer información adicional en el pronóstico y diagnóstico de esta patología ${ }^{11}$.

Es importante mencionar que el diagnóstico temprano de esta enfermedad, así como conocer los factores de riesgo predisponentes y demás aspectos epidemiológicos servirá para disminuir la morbi-mortalidad de los pacientes ${ }^{12,13}$.

La indicación para la toma de hemocultivos, es que se realice antes del inicio de la terapia antibiótica. Dependiendo de la sospecha clínica y de las condiciones

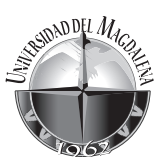


del paciente se deben incluir las botellas aerobias y/o anaerobias según el microrganismo que se sospeche, para aumentar la posibilidad de identificarlos, tomando al menos uno recogido por vía percutánea y otro recogido a través de cada catéter si se sospechara que es esta vía de acceso es la causante de la infección ${ }^{6}$.

Teniendo en cuenta que, en la ciudad de Santa Marta, no se han encontrado estudios sobre el tema, surgió el interés en desarrollar esta investigación sobre los principales aspectos epidemiológicos de la sepsis, con los pacientes sépticos de las unidades de cuidado intensivo adulto de la ciudad de Santa Marta, y conocer la realidad de esta patología de cuidado crítico en el medio.

La información obtenida en el estudio será muy útil para todo el equipo de salud de las unidades de cuidado intensivo adulto de la ciudad de Santa Marta, en aras de disminuir la morbimortalidad generada por la Sepsis, y así mismo, servirá de guía para estudios futuros y posibles proyectos investigativos sobre el tema que puede ser de gran impacto para la ciudad de Santa Marta y Colombia en general.

\section{MATERIALES Y MÉTODOS}

Estudio de tipo descriptivo, prospectivo, de corte transversal, desarrollado en el 2014, se realizó en todas las unidades de cuidados intensivos de la ciudad de Santa Marta, que corresponden a nueve, muestreo aleatorio simple por conveniencia, se tomaron 11 pacientes en 8 UCI y 12 en una de ellas. Se revisaron las historias clínicas de 100 pacientes con diagnóstico de sepsis $(\mathrm{N}=100)$. Los criterios de inclusión fueron: Pacientes con diagnóstico de sepsis, mayores de 18 años. La sepsis provenía de la comunidad y/o asociada a la atención en salud. Los criterios de exclusión fueron los pacientes con otro diagnóstico o menores de 18 años.

Entre las variables analizadas se incluyeron: Datos demográficos: edad y sexo, microorganismos causales por género y especie, foco infeccioso, letalidad.

Se realizó análisis estadístico de los datos obtenidos de la fuente de información para el respectivo análisis y conclusión del estudio; los datos obtenidos se tabularon en una tabla de Microsoft Excel para su procesamiento estadístico.

Se utilizó técnicas de análisis descriptivo de datos, ajustadas a los niveles de medición de las variables. Se realizó determinación de las frecuencias con sus valores absolutos y relativos para las variables cualitativas del estudio como el sexo de los pacientes.

\section{Declaración de aspectos éticos}

Según la Resolución No. 008430 de 1993 (4 de octubre de 1993), esta investigación es considerada de riesgo mínimo, ya que es un estudio no experimental, donde se obtendrán los datos de las historias clínicas de los pacientes. Se tuvo en cuenta la autorización por parte de la institución prestadora de salud, el consentimiento informado de los participantes; y la aprobación del proyecto por parte del Comité de Ética de la institución. Manteniéndose la privacidad de los datos recogidos ${ }^{14}$.

\section{RESULTADOS}

En el desarrollo del proyecto investigativo se muestran los siguientes resultados:

La edad promedio de los casos fue de 63, con una desviación estándar de 21,0. Edad mínima 18 años y edad máxima 99 años. La distribución por grupos de edad fue: 18-30 años (12\%), 31-40 (7 \%), 41-50 (9\%), 51-60 (6\%). 61-70 (22\%), 71-80 (24\%), 81-90 (17\%), 91-100 (3\%). (Figura 1).

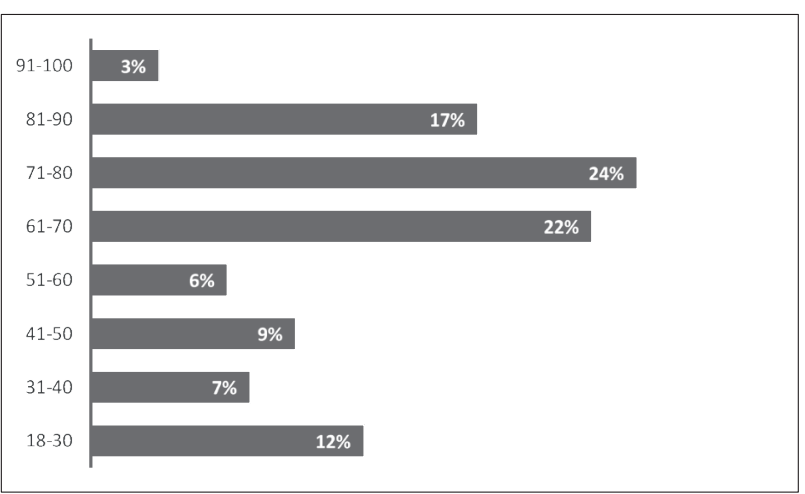

Figura 1. Distribución según grupo de edades.

En cuanto al sexo de los pacientes no hay una gran diferencia en el porcentaje entre masculino y femenino, teniendo en cuenta que el sexo femenino tuvo un $51 \%$ y el sexo masculino un $49 \%$.

De los 100 pacientes con sepsis en 45 se logró aislar algún microorganismo en los hemocultivos periféricos. 
Al analizar los datos se pudo determinar que los microorganismos prevalentes se distribuyeron así: el $76 \%$ equivale a los gérmenes Gram negativos, el $20 \%$ a los gérmenes gram positivos y solo el $4 \%$ a los hongos (Figura 2).

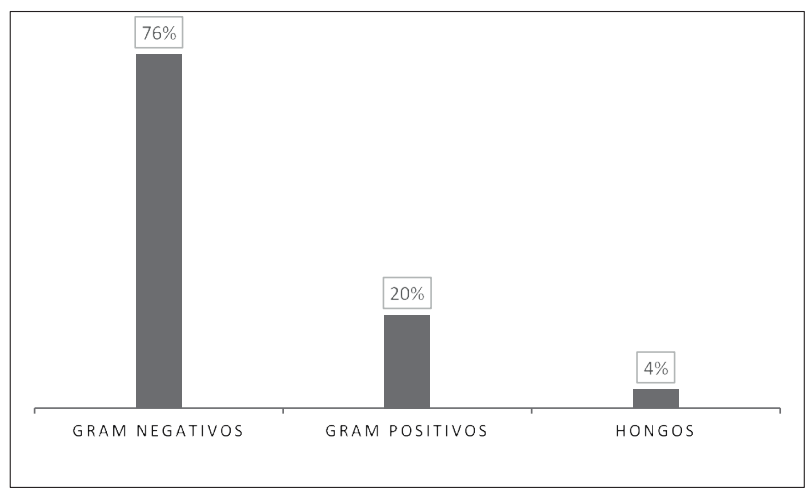

Figura 2. Microorganismos causales de sepsis.

Siendo las bacterias negativas los agentes causales predominantes, las más frecuentes fueron: Escherichia coli, Klebsiella pneumoniae spp pnuemoniae, Pseudomona aeruginosa y Serratia marcensens; entre las bacterias gram positivos el más frecuente fue el Staphylococcus aureus y los hongos que predominaron fueron las Candidas albicans y Candida haemulonii. (Figura 3).

De los100 pacientes sépticos, el principal foco primario de infección fue el de origen abdominal teniendo una frecuencia de 27 pacientes que equivalen al $30 \%$, en segundo lugar está el foco del tracto urinario con frecuencia de 20 pacientes equivalente al $22 \%$, en tercer lugar está el foco de origen de piel y tejidos blandos con frecuencia de 16 pacientes equivalente a $18 \%$, en cuarto lugar está el foco de origen pulmonar con frecuencia de 15 equivalente al $16 \%$ y con menor frecuencia están los focos de origen por catéter, sistema nervioso central e indeterminado. (Figura 4).

Al analizar la letalidad de los pacientes sépticos, que murieron mientras estaban hospitalizados en la UCI, se obtuvo como resultado: que, el $33 \%$ de los pacientes con sepsis fallecieron y $67 \%$ de los pacientes lograron sobrevivir a la enfermedad. (Figura 5).

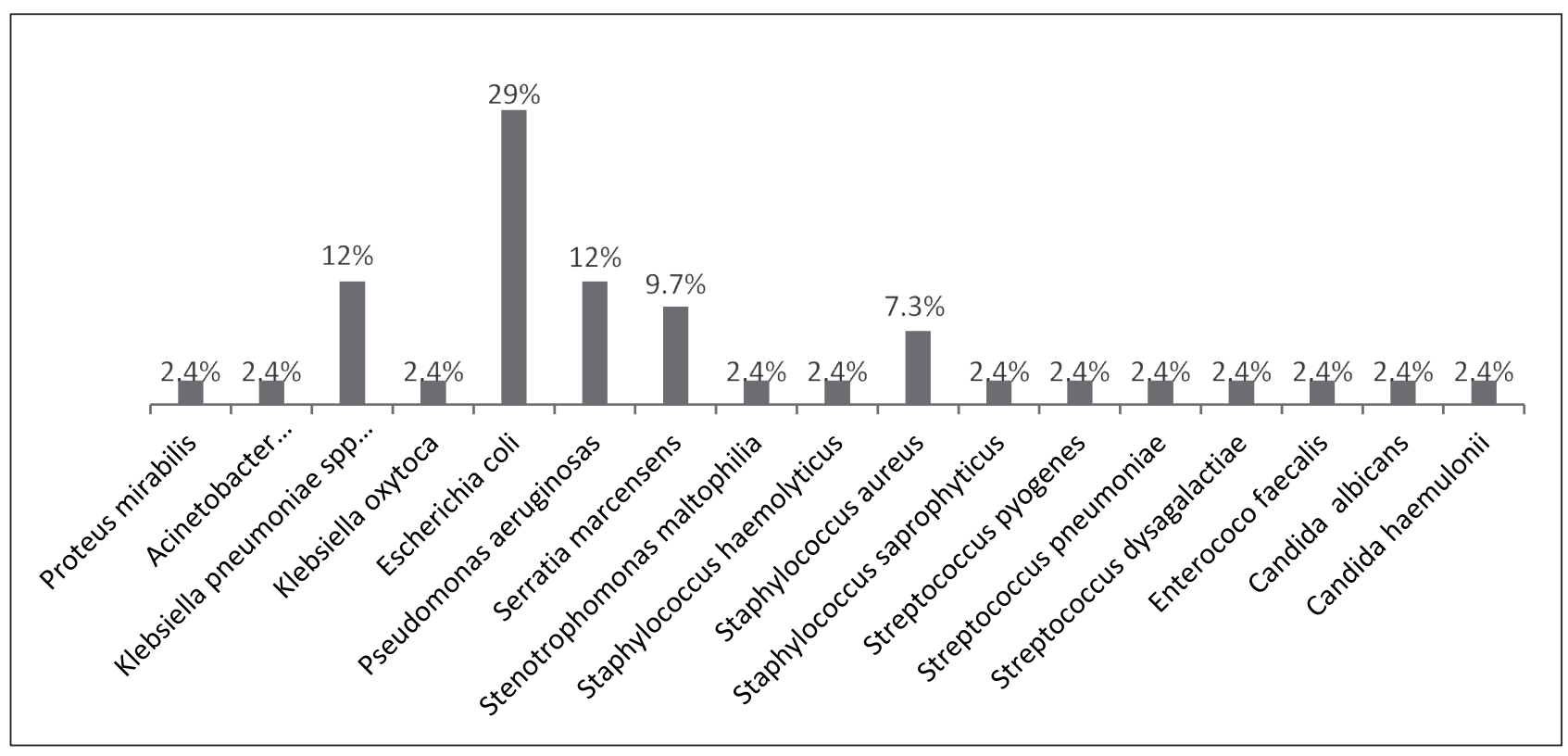

Figura 3. Microorganismos más frecuentes por género y especie.

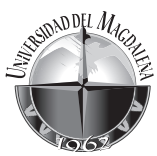




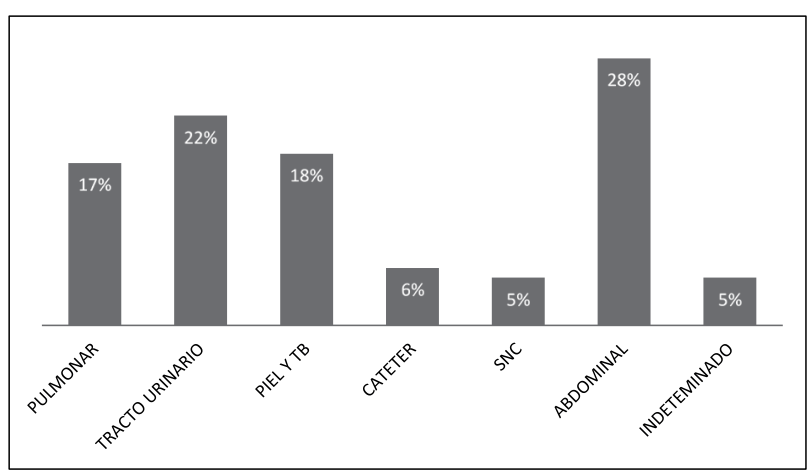

Figura 4. Focos infecciosos relacionados con sepsis.

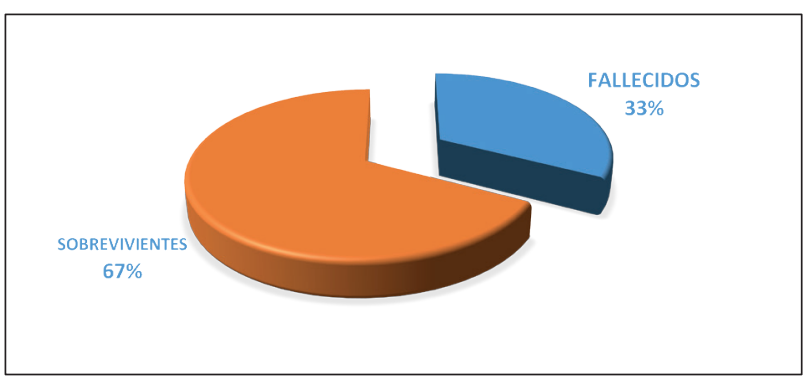

Figura 5. Letalidad de los pacientes sépticos.

\section{DISCUSIÓN}

El presente estudio da a conocer aspectos epidemiológicos de los pacientes sépticos ingresados a la UCIA.

A partir de los resultados encontrados, se puede determinar que no se han presentado diferencias notables en relación con estudios anteriores; Carbajal ${ }^{7}$ realizó una investigación en España que evidenció que, de igual modo, la prevalencia de gérmenes gramnegativos era mayor que la de Gram positivos y hongos en el desarrollo de la sepsis. También en el estudio de Espinoza et $\mathrm{al}^{15}$ los gérmenes gramnegativos fueron los más aislados. Actualmente se incorpora la genómica para identificación microbiana, Thompson et al ${ }^{16}$ utilizo la técnica Magicplex Sepsis Real-time para realizar el diagnóstico molecular y determinar el agente causal de sepsis; obteniendo también mayor incidencia de bacterias Gram negativas.

En lo que se refiere a la edad promedio de estos pacientes, difiere con el estudio de Ocampo et al ${ }^{17}$ donde esta fue de 45 años y en este estudio fue de 63. Y tampoco concuerda con que la mayoría de ellos fueron de sexo femenino; ya que en esta investigación no hubo diferencias significativas.
Así mismo, Roca y Roca ${ }^{18}$ afirma que "cualquier paciente tiene riesgo de sufrir sepsis, aunque este riesgo se incremente en los extremos de la vida (menores de 1 año y mayores de 60 años)"; en este estudio más de la mitad de los pacientes sobrepasaban los 60 años.

Con respecto al origen de la infección, el que mayor prevalencia presento fue el foco abdominal, seguido del tracto urinario, pulmonar y en menor proporción piel y tejidos blandos, focos de origen por catéter y sistema nervioso central, lo que difiere con la investigación de Mendoza $^{19}$ en el que las infecciones urinarias y las respiratorias son las más frecuentemente asociadas a sepsis; como se planteó inicialmente, sin embargo las causas subsecuentes fueron la infección de tejidos blandos; seguidas de las infecciones digestivas.

En cambio, hay una mejor concordancia con el estudio de Ocampo et al ${ }^{17}$ en el que pacientes sépticos, la mayoría de los casos el origen de la sepsis fue abdominal.

En el análisis de la frecuencia de los agentes causales, los hemocultivos permitieron establecer la etiología infecciosa y sigue siendo aún el estudio de elección para confirmar una bacteriemia en pacientes con o sin foco evidente de infección, pero por otra parte solo salieron positivos en el $45 \%$ de los pacientes diagnosticados con sepsis, lo que significa que el cultivo de la sangre tuvo un bajo rendimiento para diagnosticar sepsis. Según el estudio de Blanco et $\mathrm{al}^{20}$, dice que el rendimiento de los hemocultivos es bajo en pacientes sospechosos y diagnosticados con sepsis. El uso previo de antibióticos en los pacientes, a pesar del uso de metodología apropiada, disminuye el índice de recuperación de microorganismos.

En cuanto a la letalidad de los pacientes sépticos, Rodríguez et al ${ }^{21}$ dice que una alta proporción de pacientes admitidos a las unidades de cuidado crítico fallece, se estima que la sepsis grave o choque séptico conlleva una mortalidad del 45,6\%. En esta investigación la letalidad estuvo en un $33 \%$ difiriendo con este autor y también con el estudio de González ${ }^{22}$ cuyo porcentaje fue de 44,3\%. Esta es una enfermedad que diagnosticada y tratada a tiempo se puede lograr aumento de la supervivencia. Una mortalidad global del 33,6\% se obtuvo en el estudio de Ortiz et al ${ }^{23}$, dato que se asemeja al obtenido. Cabe resaltar que este porcentaje de mortalidad se obtuvo de la base de datos de cada UCIA, pero que se debiera realizar un estudio más profundo sobre todos los eventos patológicos que llevaron al paciente a la muerte. 
Como conclusiones se determina que La edad promedio de los casos fue de 63, En cuanto al sexo de los pacientes no hay diferencias significativas entre masculino y femenino. Los gérmenes gram negativos son los principales causantes de sepsis, siendo las bacterias más frecuentes son Escherichia Coli, Klebsiella pneumoniae, Pseudomona aureuginosa y Serratia marcenscens, Los hongos son los microrganismos de menor incidencia, siendo la Candida albicans la más encontrada y otras levaduras emergentes como la Candida haemulonii. Los focos infecciosos más relevantes asociados a sepsis son en primer lugar el abdominal seguido del tracto urinario y la piel y tejidos blandos. Esto es muy importante tenerlo en cuenta para adecuadas terapias antimicrobianas. El rendimiento de los hemocultivos es bajo en pacientes diagnosticados con sepsis, se hace necesario pensar en otras alternativas de diagnóstico como el uso de biomarcadores.

La sepsis es una enfermedad que puede ser prevenida y a nivel intrahospitalario, cada miembro del equipo interdisciplinario de salud, es responsable de esto. El lavado de manos y la aplicación de técnicas asépticas según las normas y protocolos establecidos es la principal forma de lograrlo.

\section{DECLARACIÓN SOBRE CONFLICTO DE INTERESES}

Los autores declaran no tener ningún conflicto de intereses.

\section{REFERENCIAS BIBLIOGRÁFICAS}

1. Perez M, Sánchez L. Actualización de la sepsis en adultos código sepsis. Andalucía: Creative Commons; 2015 [Accesado 10 de mayo de 2014] Disponible en http:// dspace.unia.es/bitstream/handle/10334/3418/0607_P $\%$ C3\% A9rez.pdf?sequence $=3$

2. Azkárate L, Sebastián R, Cabarcos E, Choperena G, Pascal $\mathrm{M}$, Salas E. Registro observacional choque severa sepsis / séptico prospectivo en un hospital de tercer nivel en la provincia de Guipúzcoa. 2012; 36(4):250-256 [Accesado 10 de mayo de 2014] Disponible en http://linkinghub. elsevier.com/retrieve/pii/S2173572712000756?via = sd

3. Valverde Y. Fundamentos epidemiológicos, fisiopatológicos y clínicos de la sepsis en la Población infantil. MEDISAN. 2011; 15(8):1156-1165 [Accesado en 20 de junio 2014] Disponible en http://scielo.sld.cu/pdf/ san/v15n8/san15811.pdf.

4. Pallas L. Interleukina G. Valor pronóstico de mortalidad y fracaso multiorgánico en el paciente crítico. [Tesis para la obtención del grado de doctor en Medicina y Cirugía].
Valencia: Universidad de Valencia; 2013 [Accesado el 5 de mayo 2014] Disponible en http://roderic.uv.es/ handle/10550/34696.

5. Molina F, Díaz C, Barreras L, De la Rosa G, Dennis $R$, Dueñas $C$, et al. Perfil microbiológico de la Infecciones en Unidades de Cuidados Intensivos de Colombia. 2011; 35(2):75 - 83. [Accesado en 20 de junio 2014] Recuperado de http://scielo.isciii.es/scielo. php?pid $=$ S0210-56912011000200003\&script $=$ sci_arttext

6. Phillip R, Levy M, Rhodes A, Annane D, Gerlach H, Opal S, et al. Campaña para sobrevivir a la sepsis: recomendaciones internacionales para el tratamiento de sepsis grave y choque septicémico, 2012. Revista Critical Care Medicine 2013; 36(4):250-256. [Accesado 10 de mayo de 2014] Disponible en http:// www.survivingsepsis.org/SiteCollectionDocuments/ Guidelines-Spanish.pdf

7. Carbajal J, Cayuela, A, Fernández, E, Aldabó T, Marquez J, Ortiz C, Garnacho J. Epidemiología y pronóstico tardío de la sepsis en ancianos. Revista Medicina Intensiva. 2013; 38(1):21-32. [Accesado el 24 de septiembre del 2014] Disponible en http://www.sciencedirect.com/ science/article/pii/S0210569112003774

8. Levy M, Fink M, Marshall J, Abraham E, Angus D, Cook D, et al. SCCM/ESCIM/ACCP/ATS/SIS International Sepsis Definitions Conference. Revista Intensive Care Med. 2003; 29:530-538

9. Hortensia A, Dáger A. Sepsis y trastornos relacionados. MEDISAN. 2012; 16(6): 932-948

10. Nistal j, García A, Miranda O, Gutiérrez L, Planes N, García G. Sepsis nosocomial en unidad de cuidados intensivos Polivalente. Rev Cubana de Med Militar. 2013; 42(2):191-200

11. Jiménez A, Candel F, González J. Utilidad de los biomarcadores de inflamación e infección en los servicios de urgencias. Enferm Infecc Microbiol Clin. 2014;32(3):177-190 [Accesado 20 agosto de 2014] Disponible en http://www.semes.org/revista/ vol24_5/2.pdf.

12. González R, López O, Mestre P, Sosa O. Caracterización epidemiológica de la sepsis en la Unidad de Cuidados Intensivos del hospital: comandante pinares. Rev Cub Med Int Emerg. 2013; 12(4)

13. Reina-Figueroa J, Richardson López CV, Vidal VP. De las definiciones, las vacunas y la identificación del paciente séptico en Pediatría. Rev Panam Salud Pública. 2010; 27(6): 469-470.

14. Colombia. Ministerio de salud. Resolución No. 008430. Por la cual se establecen las normas científicas, técnicas y administrativas para la investigación en salud. (4 de octubre de 1993). [Accesado el 20 de septiembre del 2014] Disponible en https://www.unisabana.edu.co/ fileadmin/Documentos/Investigacion/comite_de_etica/ Res_8430_1993_-_Salud.pdf 
15. Espinosa F, Hart M, Halley M, Zamora R. Control multidisciplinario de la infección nosocomial en un hospital de nivel terciario. Revista Cubana de Medicina. 2011; 50(1): 40-48.

16. Thompson M, Rosas G, Lara M, Merino J, Moreno H, Morán E, et al. Diagnóstico molecular de sepsis polimicrobiana en pacientes internados en la unidad de cuidados Intensivos. Rev. Esp Médico Quir. 2014; $19(1): 52-61$.

17. Ocampo M, Lugo C, Cordero L, Blanco G. Caracterización epidemiológica de la sepsis en el Hospital General de San Cristóbal de Las Casas. Revista CENIC. Ciencias Biológicas, 2010; 41:1-10. [Accesado el 22 de septiembre del 2014] Disponible en http://www.redalyc.org/ articulo.oa?id $=181220509010$

18. Roca B, Roca I. Sepsis y síndromes relacionados Servicio de Medicina Interna e Infecciones. Hospital General de Castellón. Universidad de Valencia. Revista médica Univ Navarra España, 2008; 52(4) 3-14

19. Mendoza A. Causas frecuentes de sepsis en pacientes adultos del servicio de urgencias del hospital general de zona no.8 Gilberto flores izquierdo del instituto mexicano del seguro social. [Tesis para obtener el título especialidad en urgencias medico quirúrgicas]. México: Instituto
Politécnico Nacional; 2011 [Accesado el 5 de mayo 2014] Disponible en http://www.repositoriodigital.ipn.mx/ bitstream/handle/123456789/12617/TESIS \%20-\%20 ARFAX \% 20ISRAEL \% 20MENDOZA \% 20CAMARENA. pdf? sequence $=1$

20. Blanco M, Scandizzo E, González Y, Pestana L, Albarenque F. Frecuencia de aislamientos microbiológicos en hemocultivos. Rev Hsp. del Cruce Argentina, 2011;10:813 [Accesado el 22 de septiembre del 2014] Disponible en http://www.hospitalelcruce.org/revis10/nota2.pdf

21. Rodriguez F, Barrera L, De la Rosa G, Dennis R, Dueñas C, Granados M, et al. The epidemiology of sepsis in Colombia: A prospective multicenter cohort study in ten university hospitals Crit Care Med. 2011;39(7):1675-82.

22. Gonzales M. Relación entre mortalidad hospitalaria y sepsis durante 15 años. Hospital Clínico Quirúrgico Docente Manuel Fajardo. Rev Científica Cubana. 2011; [Accesado el 22 de septiembre del 2014] Disponible en http://www.bvs.sld.cu/revistas/mie/vol10_2_11/ mie04211.html

23. Ortiz G, Dueñas C, Rodríguez F, Barrera L, De La Rosa $\mathrm{G}$, Dennis R, et al. Epidemiology of sepsis in Colombian intensive care units. Revista Biomédica. Colombia, 2014;34(1):40-7.

Para citar este artículo: Pertuz-Meza Y, Pérez-Quintero C, Pabón-Varela Y. Aspectos epidemiológicos de la sepsis, en unidades de cuidados intensivos Santa Marta, Colombia. Duazary. 2016 julio; 13 (2): 126 - 132 